\title{
EDITORIAL
}

\section{SOBRE LA METODOLOGÍA CUALITATIVA (*)}

Se me atribuye, por ejemplo, la invención del grupo de discusión. Cuando en esa invención participaron a la par que yo, muchos: Alfonso Ortí, Paco Pereña, Jose Luis de Zárraga y Ángel de Lucas, muy especialmente .

Jesús Ibáñez

\section{Cristina Pérez Andrés}

Comité de redacción de la Revista Española de Salud Pública. Subdirección General de Promoción de la Salud y Epidemiología. Dirección General de Salud Pública. Ministerio de Sanidad y Consumo.

(*) Este editorial está dedicado a la memoria de Juan Blanco, maestro de Filosofía, sin cuyas enseñanzas no podría haber sido escrito.

Hace ahora diez años, a finales de agosto de 1992, moría Jesús Ibáñez, gran teórico y práctico de la investigación social en España, concretamente de la metodología estructural o cualitativa. Licenciado en Ciencias Políticas, aunque lo suyo era la filosofía, se aficionó a la sociología y trabajó en el Instituto de Opinión Pública y en el Instituto de Estudios Políticos, donde durante un seminario, en el que él mismo participaba, se gestó la investigación social en España. En 1958, tras unos años de estancia en la cárcel por motivos políticos, se le propuso fundar un Instituto de Mercado y así nació ECO, la primera empresa española de investigación. Allí conoció a Alfonso Ortí, posteriormente en CEISA a Ángel de Lucas y José Luis de Zárraga y juntos fundaron ALEF. Con la colaboración del psicoanalista Francisco Pereña, entre todos inventaron el grupo de discusión ${ }^{1}$. Con esta técnica trabajaron haciendo investigaciones de mercado y poniendo a la cabeza del mismo los productos de las firmas que les contrataban. En 1969 Jesús Ibáñez se incorporó a la Universidad, obteniendo la plaza de profesor titular tras una oposición cuya memoria se recoge en su libro $\mathrm{Del}$ algoritmo al sujeto ${ }^{2}$. Más tarde en su tesis doctoral Más allá de la sociología $a^{3}$, funda- mentó el grupo de discusión desde el punto de vista teórico, metodológico y epistemológico. En 1982 obtuvo la cátedra de Técnicas de Investigación Social, en la que junto con Lluis Martín Santos y Ángel de Lucas se formaron en metodología cualitativa numerosos alumnos que dieron lugar a la Escuela Cualitativa de Madrid.

En palabras de Jesús Ibáñez, las llamadas técnicas cuantitativas investigan el sentido producido (los hechos). La técnica del grupo de discusión investiga el proceso de producción de sentido, que no es más que la reproducción de la unidad social de sentido, y en ello reside su valor técnico ${ }^{1}$. Y lo hacen siguiendo el camino inverso al de su producción, es decir obteniendo discursos cuyo análisis e interpretación llevan al origen y al proceso de formación de las unidades de sentido que aparecen en el contenido manifiesto de los mismos.

Desde los primeros tiempos de su existencia, el ser humano se ha relacionado con la naturaleza y ha explicado sus leyes mediante un proceso de simbolización. Con su capacidad de hablar adquirió la de la representación mental y por lo tanto la de interiorizar e inter- 
pretar, dando lugar a la abstracción (pensamiento) y con ella al conocimiento ${ }^{4}$. Así surgió primero el pensamiento mágico con el que el ser humano daba una explicación a los fenómenos que aparecían ante él. Con él se construyeron los mitos y las cosmogonías, que generaban un conocimiento colectivo sobre los orígenes del Universo y los fundamentos de sus culturas y relaciones sociales. Este pensamiento mágico, tanto más presente cuanto menos avanzada sea una civilización, ha ido siendo sustituido por el pensamiento religioso, filosófico y el científico a medida que la humanidad se ha ido desarrollando social y culturalmente. Sin embargo, también en las sociedades avanzadas se sigue dando, sobre todo durante la infancia pero también en la vida adulta, siendo la base del pensamiento y del lenguaje tradicional, los cuales utilizamos para representarnos el mundo y la sociedad en que vivimos, así como para comunicarnos en nuestra vida cotidiana. Corresponde a una interpretación que los sujetos hacen por analogía entre lo que perciben y la explicación que le dan, y se hace a través de la elaboración de símbolos (metáforas y metonimias) que dan un sentido a lo que hasta entonces no lo tenía, produciendo un conocimiento que disminuye la angustia del individuo en particular y la de la humanidad en general ${ }^{5}$. La interpretación de estas analogías contribuye al conocimiento de sus orígenes y al del proceso de su formación y, por tanto, al conocimiento de su sentido. Como ya hemos dicho, es la facultad que tiene el ser humano de hablar y por lo tanto de pensar y elaborar a partir de un «material primario» otro más complejo, lo que le permite efectuar este proceso de simbolización. Aunque entre los animales se identifican «lenguajes» (cuyo código ha sido ideado por el ser humano), éstos no sirven para elaborar ideas ni analogías ni, por lo tanto, sentido. Dada la naturaleza polisémica del lenguaje del ser humano, éste puede sacar más de un sentido de los significantes y los significados utilizados en el habla $^{6}$, algo que los animales jamás serán capaces de hacer, por lo que nunca podrán elaborar un chiste ni una representación, de la misma forma que son incapaces de elaborar los alimentos que van a ingerir, no solo porque no manejan el fuego, tampoco harán nunca una ensalada con ellos.

Por ello podemos decir que lo que convierte al ser humano en diferente del resto de los animales es su lenguaje y con él la facultad de simbolización. Entendiendo así las cosas podemos también comprender que investigando el lenguaje y el habla de los sujetos se pueda llegar a la explicación de múltiples aspectos de la realidad planteados como objeto de estudio. Así, la investigación cualitativa utiliza como datos las representaciones y los discursos obtenidos en condiciones rigurosamente diseñadas para llegar, mediante el análisis y la interpretación de las unidades de sentido (estructuras semánticas) identificadas en ellos, al origen y significación de las analogías (metonimias y metáforas) utilizadas para elaborarlas, lo que hace siguiendo el camino inverso al de su formación, es decir, al del proceso de la simbolización.

Sabiendo que el habla de un sujeto depende de su subjetividad y de la intersubjetividad con la de otros, las cuales a su vez están condicionadas por el contexto cultural, social e histórico en el que se desarrolla, se tendrá claro por qué es imprescindible el conocimiento de aquellas disciplinas relacionadas con el conocimiento de la cultura, de la sociedad y de la historia para investigar con el lenguaje y el habla de los sujetos y con los discursos y representaciones a que dan lugar. Así, para practicar la investigación cualitativa son necesarios conocimientos sobre la subjetividad y el inconsciente (psicoanálisis), los significantes y los significados de las palabras y los signos (lingüística, semiología), el sentido de los mismos (semántica), la interpretación de los símbolos (hermenéutica), la cultura (antropología), la percepción de la realidad (fenomenología) y sobre la sociedad (sociología). La metodología cualitativa es, pues, una forma multidisciplinar de acercarse al conocimiento de la realidad social. 
En el terreno de las ciencias sociales se pueden estudiar tanto los hechos (desde el punto de vista de la frecuencia de su presentación, etcétera) de lo cual se encarga la metodología cuantitativa, como el sentido y la significación de las representaciones sociales y los discursos ${ }^{7-11}$, de lo cual se encarga la metodología cualitativa, esta última a través del análisis del contenido manifiesto de los mismos para, mediante la interpretación, obtener el oculto o latente. Dado que ninguna actividad humana escapa al lenguaje y al habla, podremos emplear la metodología cualitativa para investigar cualquier fenómeno relacionado con la realidad social, entre ellos la salud y la enfermedad.

Aunque la metodología más frecuentemente utilizada para investigar en ciencias de la salud es la cuantitativa o epidemiológica, en la actualidad es posible afirmar que de la investigación cualitativa, cuando menos, ha oído hablar la mayor parte de las personas que trabajan en el sector sanitario. Sin embargo, dado que las disciplinas que la conforman no son frecuentemente conocidas, existen en su aplicación algunos errores que la invalidan, y sobre ella algunos mitos que convendría aclarar.

Se puede decir que ha sido recientemente, en la década de los 90, cuando tanto en España $^{12}$ como en el ámbito anglosajón se ha empezado a promocionar la utilización de las técnicas cualitativas en el ámbito de la salud. Y como aquí estamos acostumbrados a dudar de lo nuestro, dando por hecho que lo anglosajón es necesariamente mejor, gran parte de los profesionales sanitarios que practican metodología cualitativa la han aprendido fundamentalmente de los autores extranjeros, ignorando la existencia de una escuela en nuestro país y los textos que la respaldan.

Así, en el ámbito sanitario se practica sobre todo el llamado grupo focal, que aunque tiene semejanzas con el grupo de discusión también tiene importantes diferencias que le distancian de él. La principal de ellas es que el grupo focal no prescinde nunca de un guión de preguntas relacionadas con el objetivo de la investigación que la persona que modera el grupo dirige a sus participantes, lo que convierte a este tipo de reunión grupal en una entrevista semiestructurada y dirigida, que busca con preguntas las respuestas del grupo (como en la metodología cuantitativa). En realidad estas preguntas son unidades de sentido prefijadas por las personas que realizan la investigación. Por el contrario, al realizar un grupo de discusión o una entrevista en profundidad se asume que los participantes en el mismo tienen las respuestas a los objetivos que en la investigación se han planteado, pero, ¡muy importante!, no saben que la tienen. El moderador, con su intervención inicial debe producir el discurso del grupo, dejándole hablar sin dirigirle preguntas directas que siempre serán respondidas a nivel racional o consciente, y busca el concurso preconsciente de los participantes. Tras obtener el discurso, la labor del investigador es llevar a cabo el proceso contrario al de la formación de las unidades de sentido que el grupo ha expresado y que el investigador encuentra en su análisis, para interpretar su origen y con él su sentido (contenido latente). Con ello podremos conocer las representaciones sociales sobre la salud ${ }^{13}$, sobre la enfermedad en general o de cualquiera de ellas en particular ${ }^{14}$, sobre las relaciones entre personas enfermas y los profesionales sanitarios que las atienden ${ }^{15}$, la aceptación por los profesionales sanitarios de nuevas formas de gestión $^{16}$, la percepción del riesgo ${ }^{17}$, las representaciones sociales sobre la donación de sangre $^{18}, \ldots$ sin haber intervenido en el discurso de los sujetos que las aportan.

Es también un error decir y escribir que la investigación cualitativa en salud procede de las ciencias sociales, porque no es que proceda de ellas (la epidemiología sí), si no que es la aplicación de la Ciencia Social al estudio de la salud y de la enfermedad, las cuales no dejan de ser construcciones discursivas formadas a partir de los diferentes discursos sociales. Apellidar a la metodolo- 
gía cualitativa con el término «salud», aproximándola así, de manera artificial, a las Ciencias Naturales, ha llevado a considerar la investigación cualitativa en salud como procedente de las Ciencias Sociales pero independiente y distinta de ellas, dando lugar a la elaboración de una serie de criterios de calidad específicos que no son más que el reflejo de pensar que la investigación cualitativa aplicada a la salud tiene permiso para existir sólo después de demostrar con criterios objetivos que, por subjetiva que sea, es válida. Por algunos de los Originales recibidos en la redacción de la Revista Española de Salud Pública para participar en este monográfico se deduce que estos criterios de calidad se han convertido en la biblia de la investigación cualitativa y han dado lugar a pensar que cualquier estudio que cumpla con dichos criterios está bien hecho, por mucho que carezca de un buen diseño y del análisis e interpretación inherentes a la metodología cualitativa. Y al contrario, que una investigación cualitativa bien realizada desde el punto de vista del diseño y del análisis y la interpretación no es válida si carece de la explicitación de que cumple con los criterios de calidad que alguien ha formalizado para la investigación cualitativa en salud. Es por eso que creo que convendría dejar de utilizar la expresión «Investigación Cualitativa en Salud» y seguir denominándola, simplemente, Investigación Cualitativa. Es la salud la que tiene que acercarse a la ciencias Sociales para dejarse estudiar por ellas, no la metodología cualitativa la que debe «contaminarse» de los criterios de las ciencias naturales al añadirle el término «salud» a su denominación. Uno de estos criterios (feed-back con los participantes) exige volver a las mismas personas que formaron el grupo para ver si reconocen como resultados las cosas que en él se dijeron. Dado que los participantes en las técnicas cualitativas tienen la respuesta a los objetivos de la misma sin saber que la tienen, y por ello la labor del investigador es elaborarla a partir del contenido de los discursos, si se vuelve al grupo para preguntarle si reconocen los resultados cabe la posibilidad de que los parti- cipantes en el mismo no los identifiquen, ya que lo que se les debería presentar como resultados son los elaborados a partir del contenido latente de su discurso, del cual, en principio, no han sido conscientes. Por otra parte, si la investigación está bien hecha, el grupo no es el único que debería reconocer los resultados como propios, si no todo el contexto social del que se ha extraído la muestra estructural que lo representa.

Otro error puede ser plantear que un estudio cualitativo es de tipo etnográfico o fenomenológico. Como hemos dicho antes, la metodología cualitativa integra una perspectiva multidisciplinar para conocer una realidad social dada y, en general, de ningún estudio se podrá decir si es de un tipo u otro, dado que todas las disciplinas que configuran la investigación cualitativa deberían estar presentes a la hora de hacer un buen diseño y, sobre todo, un buen análisis y una buena interpretación.

Pero, en mi opinión, el error más importante que se comete es el de confundir el análisis y la interpretación con la mera clasificación y estructuración de la información contenida en el discurso manifiesto, llegando a unas conclusiones que carecen del sentido que se debería haber buscado. Ello es lo que ha ocurrido con la mayoría de los trabajos originales que se han recibido en la redacción de la Revista Española de Salud Pública para participar en este número monográfico sobre metodología cualitativa, los cuales, tuvieran o no un buen diseño, carecían del proceso esencial de análisis del contenido manifiesto de los discursos para obtener mediante su interpretación el contenido latente y a partir de él elaborar los resultados. En cambio, los que se presentan en estos trabajos consisten en la simple estructuración y ordenación de la información que contienen las transcripciones en base, además, a una estructura prefijada (guión de la entrevista). En metodología cualitativa, las unidades de sentido no deben fijarse $a$ priori antes de realizar los grupos o las entrevistas, sino que se encuentran en los discursos y en 
las construcciones sociales mediante el análisis de los mismos, y son las que posteriormente han de servir para realizar la interpretación y si con el contenido manifiesto no se ha hecho esta labor no hay metodología cualitativa. Este es uno de los motivos por el que no se pueden clasificar las técnicas de consenso dentro de esta metodología, dado que en ellas no hay ninguna labor de interpretación ${ }^{19}$.

En cuanto a los mitos que existen sobre la investigación cualitativa, uno muy frecuente es el de creer que sólo sirve como paso previo a la construcción de un cuestionario, que luego utilizará la metodología epidemiológica para medir, es decir, objetivar, y que además es ese cuestionario el que le da validez a la metodología cualitativa utilizada. Al contrario de esta creencia, los resultados y conclusiones de una investigación realizada con metodología cualitativa, si la muestra social ha sido bien elegida, han de contener las representaciones sociales que existen en el contexto social del que ha sido extraída. Bueno sería que cada vez que se diseña un cuestionario, porque hay necesidad de medir el objeto de estudio, se hubiera realizado previamente una investigación cualitativa para saber qué hay que preguntar, pero eso no quiere decir que toda técnica cualitativa deba seguirse de la construcción de un cuestionario. La mayoría de investigaciones cualitativas que se hacen, en el ámbito de la salud o fuera de él, no precisan de la cuantificación de lo encontrado y sus informes son válidos para plantear las medidas correctoras de los problemas que se han estudiado con ellas.

Otro mito sobre la metodología cualitativa es que es propia del ámbito de la promoción de la salud, suponemos que porque es la que trabaja con la población «sana». Sin embargo, con la metodología cualitativa se pueden investigar objetivos relacionados con todos los aspectos de la salud y la enfermedad, relativos a la planificación y gestión sanitaria, a las vivencias de las personas aquejadas por una enfermedad o por deter- minados factores de riesgo, a las relaciones entre los profesionales sanitarios y los sujetos a los que atienden, etcétera. Aunque también es un mito pensar que la metodología cualitativa es un trabajo propio del terreno de la enfermería, en este caso tenemos que reconocer el mayor esfuerzo que han hecho estos profesionales para aprender y aplicar esta metodología. Buen ejemplo de ello es la revista Index de enfermería, en la que la mayor parte de los trabajos que se publican están realizados con metodología cualitativa. Ello merece nuestra felicitación, sin dejar de insistir, como en el punto anterior que se puede aplicar a cualquier nivel del ámbito de la salud y la enfermedad en el que interese conocer la experiencia de sus protagonistas como sujetos. En cualquier caso, parece que ambos mitos están relacionados entre sí y los dos a su vez con la opinión que de la metodología cualitativa se tiene, porque así como el trabajo de promoción de la salud está desprestigiado con respecto al de la curación de la enfermedad, el trabajo de enfermería lo está con respecto al trabajo de los médicos, de la misma forma que la metodología cualitativa es considerada menos válida que la cuantitativa.

Un cuarto mito tiene que ver con la valoración que los positivistas hacen tanto sobre la forma de obtener los resultados en metodología cualitativa, equiparando interpretación con invención, como sobre la imposibilidad de inferencia de los mismos. Achacar a la subjetividad del investigador y a la de los sujetos que forman la muestra (cuando a ella no puede renunciar nadie) la falta de validez de los resultados de la metodología cualitativa es, entre otras cosas, negar que esta subjetividad también existe en los métodos cuantitativos, tanto por parte del investigador (cuando elige el tema de investigación, cuando elabora el cuestionario y cuando realiza el informe de resultados) como de los sujetos investigados (cuando deciden participar o no y cuando responden a las preguntas). De manera que descalificar la metodología cualitativa por basarse en la subjetividad que nos constituye es descalifi- 
car a su vez la cuantitativa, ya que por más que lo pretendiera nunca nadie podrá prescindir de la subjetividad.

Por otra parte, es necesario no perder de vista que la inferencia estadística que la epidemiología utiliza para generalizar a la población los resultados encontrados en sus muestras, no deja de ser el cálculo de una probabilidad, y así, el conocimiento obtenido en una muestra de individuos tampoco se puede asegurar que se dé en la población de referencia sino en términos de posibilidad, algo que sólo podríamos superar repitiendo las medidas en todas y cada una de las personas que forman la población a la cual se infieren los resultados encontrados. $\mathrm{Y}$ es a causa de esta forma de inferir los resultados por parte de la metodología cuantitativa que también se rechaza la validez de los resultados de la investigación cualitativa, debido a la falta de representatividad estadística de las personas que forman sus muestras. $\mathrm{La}$ representatividad de la muestra cualitativa es estructural, es decir representa la estructura de las relaciones que se dan en el grupo social que se estudia, y de la misma forma que un investigador con metodología cualitativa no puede achacar a un estudio cuantitativo falta de validez porque carece de esta representatividad estructural (dado que no hay relaciones entre los individuos estudiados con metodología cuantitativa), el investigador que utiliza la metodología cuantitativa tampoco puede hacer lo contrario. Son dos metodologías diferentes porque trabajan con «géneros» distintos (las separa la misma distancia que separa la épica, o narración de los hechos, de la tragedia, o puesta en escena de las acciones a través del lenguaje), y ninguna de las dos puede ser autoreferente a la hora de criticar a la otra. En cualquier caso, tanto los partidarios de la investigación cualitativa, como los de la cuantitativa, e incluso los que entendemos que ambas son necesarias, debemos ser conscientes de que con ninguna de ellas, ni juntas ni por separado, podremos llegar nunca a la totalidad del conocimiento de ninguna realidad, sólo podremos acercarnos más a ella, pero nunca estaremos en posesión de su conocimiento absoluto, por mucho que utilicemos la ciencia, la técnica o el pensamiento. Con cada una obtendremos siempre una imagen cubista del aspecto de la realidad estudiado, modelos para seguir avanzando. Es por ello que personalmente no soy partidaria de decir que las perspectivas cuantitativas y cualitativas son complementarias, en todo caso entre ambas podrán sumar conocimiento, pero nunca completarán el de ninguna realidad.

Para concluir, dado que en nuestro medio existen dos modalidades de investigación cualitativa, la procedente de nuestro país y la del ámbito anglosajón, este monográfico incluye trabajos basados en una o en otra, por lo que los lectores encontrarán criterios diferentes en sus contenidos. Sin embargo, personalmente animo a las personas interesadas en la metodología cualitativa, a leer los textos de Jesús Ibáñez ${ }^{1-3,20,21}$, Alfonso Ortí 17,9 , Fernando Conde $8,22,23$, Francisco Pereña ${ }^{24,25}$, Anselmo Peinado ${ }^{26}$, Luis Enrique Alonso ${ }^{27}$ y un largo etcétera de investigadores, así como también animo a aprender a realizar una investigación cualitativa que no precisa de guiones de preguntas o unidades de sentido prefijadas, porque lo que interesa en metodología cualitativa no es buscarlas sino encontrarlas.

Presentamos así este número monográfico de la Revista Española de Salud Pública dedicado a la Investigación Cualitativa. La acogida de su edición fue realmente positiva a juzgar por el número de artículos originales que se recibieron para participar, treinta y cuatro, de los cuales tres eran de Andalucía, dos de Castilla la Mancha, dos de Cataluña, uno de Extremadura, nueve de Madrid, seis de la Comunidad Valenciana, uno de Islas Baleares, uno de Murcia, uno de Navarra, uno de Argentina, dos de Colombia, dos de México y uno de Perú. De ellos se publican cinco de Madrid, uno de Cataluña, uno de Islas Baleares, dos de la Comunidad Valenciana, y uno de Navarra. 
Como miembro del Comité de Redacción quiero manifestar nuestro agradecimiento a todas las personas que han participado y colaborado en la edición de este número, incluyendo a los autores y autoras de las Colaboraciones Especiales, que con sus aportaciones contribuirán al conocimiento de la metodología cualitativa y a su promoción y desarrollo en el ámbito de la salud. A los autores y autoras que enviaron sus Originales, hayan sido o no aceptados para su publicación. Muy especialmente agradecemos su trabajo a todos los evaluadores y evaluadoras que han revisado los artículos, cuya relación figura al final de este número, algunos de cuyos informes merecerían ser publicados. Su número demuestra que no hay ninguna dificultad para encontrar personas que evalúen trabajos de investigación sobre la salud realizados con metodología cualitativa cuando éstos se dirigen a una revista de salud pública, por lo que también animamos a los lectores de este editorial a que continúen enviándonos los trabajos realizados con esta metodología. Próximamente coordinaremos un número monográfico sobre metodología epidemiológica y esperamos que esa nueva experiencia sea tan grata como ha sido esta primera.

\section{BIBLIOGRAFÍA}

1. Ibáñez J. Autobiografía. (Los años de aprendizaje de Jesús Ibáñez). Anthropos 1990; núm. 113: 9-25.

2. Ibáñez J. Del algoritmo al sujeto. Madrid: Siglo XXI; 1985.

3. Ibáñez J. Más allá de la sociología. El Grupo de Discusión: teoría y crítica Madrid: Siglo XXI; 1986.

4. Argullol R. Tres miradas sobre el arte. Madrid: Destino; 2002.

5. Freud S. Introducción al psicoanálisis. Madrid: Alianza Editorial; 1967. p. 411.

6. Guiraud P. La significación: forma y sustancia del signo. En: La semiología. México: Siglo XXI; 1991. p. 33-60.
7. Ortí A. El proceso de investigación de la conducta como proceso integral: complementariedad de las técnicas cuantitativas y de las prácticas cualitativas en el análisis de las drogodependencias. En: Las drogodependencias: perspectivas actuales. Primer Encuentro Nacional sobre Sociología y Drogodependencias. Madrid: Ilustre Colegio Nacional de Doctores y Licenciados en Ciencias Políticas y Sociología; 1991. p. 150-201.

8. Conde F. Una propuesta de uso conjunto de las técnicas cuantitativas y cualitativas en la Investigación Social. El isomorfismo de las dimensiones topológicas de ambas técnicas. Rev Esp Invest Social 1987; 39: 213-24.

9. Ortí A. La apertura y el enfoque cualitativo o estructural: la entrevista abierta y la discusión de grupo. En: El análisis de la realidad social. Métodos y Técnicas de Investigación. Madrid: Alianza Universidad; 1990. p. 171-203.

10. Ibáñez J. Perspectivas de la Investigación Social: el diseño en las tres perspectivas. En: El análisis de la realidad social. Métodos y Técnicas de Investigación. Madrid: Alianza Universidad; 1990. p. 49-83.

11. Beltrán M. Cinco vías de acceso a la realidad social. En: El análisis de la realidad social. Métodos y Técnicas de Investigación. Madrid: Alianza Universidad; 1990. p. 17-47.

12. Gil Nebot M, Estrada Ballesteros C, Pires Alcalde ML y Aguirre Martín-Gil R. La investigación cualitativa y la promoción de la salud en la Comunidad de Madrid. Rev Esp Salud Publica 2002; 76: 451-459.

13. Consejería de Sanidad y Servicios Sociales. Las Representaciones Sociales sobre la salud de los jóvenes madrileños. Documentos Técnicos de Salud Pública n. ${ }^{\circ}$ 45. Madrid: Consejería de Sanidad y Servicios Sociales; 1998.

14. Consejería de salud. Actitudes ante el asma. Los asmáticos y los profesionales opinan. Madrid: Consejería de salud; 1994. Documentos técnicos de salud pública, núm. 17.

15. Consejería de salud. Discurso del personal sanitario de la Comunidad de Madrid en torno a la infección por el VIH/Sida. Madrid: Consejería de salud; 1992. Documentos técnicos de salud pública, núm. 11.

16. Pérez Andrés C. Aceptación del contrato programa por el personal sanitario de la atención primaria de Madrid: un estudio cualitativo. Rev Sanid Hig Pública 1995; 69: 79-88. 
17. Consejería de salud. Los accidentes infantiles en la Comunidad de Madrid: Percepción de los padres del riesgo de accidente. Madrid: Consejería de salud; 1993. Documentos técnicos de salud pública, núm. 35 .

18. Garay Uriarte A, Iñiguez Rueda L, Martínez González M, Muñoz Justicia J, Pallarès Segura S

y Vázquez Sixto F. Evaluación cualitativa del Sistema de recogida de sangre en Cataluña. Rev Esp Salud Pública 2002; 76: 437-450.

19. Pérez Andrés C. ¿Deben estar las técnicas de consenso incluidas entre las técnicas de investigación cualitativa? Rev Esp Salud Pública 2000; 319-21.

20. Ibáñez J. El regreso del sujeto. La investigación social de segundo orden. Madrid: Siglo XXI; 1994.

21. Ibáñez J. Por una sociología de la vida cotidiana. Madrid: Siglo XXI; 1994.

22. Conde Gutiérrez F. Las perspectivas metodológicas cualitativas y cuantitativas en el contexto de la Historia de las Ciencias. En: Métodos y técnicas de investigación en Ciencias Sociales. Delga- do JM y Gutiérrez J editores. Madrid: Síntesis Psicológica; 1994. p. 53-68.

23. Conde Gutiérrez F. Procesos e instancias de reducción/formalización de la multidimensionalidad de lo real: procesos de institucionalización/reificación social en la praxis de la investigación social. En: Métodos y técnicas de investigación en Ciencias Sociales. Delgado JM y Gutiérrez J editores. Madrid: Síntesis Psicológica; 1994. p. 100-119.

24. Pereña F. Hablar es un equívoco. Anthropos 1990; núm 113:56-8.

25. Pereña F. Formación discursiva, semántica y psicoanálisis. En: Métodos y técnicas de investigación en Ciencias Sociales. Delgado JM y Gutiérrez J editores. Madrid: Síntesis Psicológica; 1994. p. $465-479$

26. Canales M, Peinado A. Grupos de discusión. En Métodos y técnicas de investigación en Ciencias Sociales. Delgado JM y Gutiérrez J editores. Madrid: Síntesis Psicológica; 1994. p. 287-316.

27. Alonso LE. La mirada cualitativa en sociología. Madrid: Fundamentos; 1998. 